\title{
Genome-wide profiling of long non-coding RNA expression patterns in the EGFR-TKI resistance of lung adenocarcinoma by microarray
}

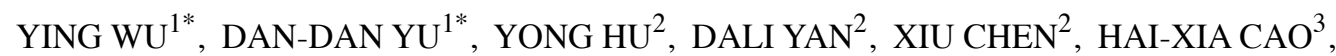 \\ SHAO-RONG YU ${ }^{3}, Z_{\text {ZHUO WANG }}^{3}$ and JI-FENG FENG ${ }^{2}$
}

${ }^{1}$ The First Clinical School of Nanjing Medical University; ${ }^{2}$ Department of Chemotherapy, Nanjing Medical University Affiliated Cancer Hospital Cancer Institute of Jiangsu Province; ${ }^{3}$ The Fourth Clinical School of Nanjing Medical University, Nanjing, Jiangsu 210009, P.R. China

Received December 20, 2015; Accepted January 27, 2016

DOI: $10.3892 /$ or.2016.4758

\begin{abstract}
Mutations in the epidermal growth factor receptor (EGFR) make lung adenocarcinoma cells sensitive to EGFR tyrosine kinase inhibitors (TKIs). Long-term cancer therapy may cause the occurrence of acquired resistance to EGFR TKIs. Long non-coding RNAs (lncRNAs) play important roles in tumor formation, tumor metastasis and the development of EGFR-TKI resistance in lung cancer. To gain insight into the molecular mechanisms of EGFR-TKI resistance, we generated an EGFR-TKI-resistant HCC827-8-1 cell line and analyzed expression patterns by 1 cRNA microarray and compared it with its parental HCC827 cell line. A total of 1,476 lncRNA transcripts and 1,026 mRNA transcripts were dysregulated in the HCC827-8-1 cells. The expression levels of 7 chosen lncRNAs were validated by real-time quantitative PCR. As indicated by functional analysis, several groups of lncRNAs may be involved in the bio-pathways associated with EGFR-TKI resistance through their cis- and/or trans-regulation of protein-coding genes. Thus, lncRNAs may be used as novel candidate biomarkers and potential targets in EGFR-TKI therapy in the future.
\end{abstract}

\section{Introduction}

Lung cancers with epidermal growth factor receptor (EGFR) gene mutations are a well-understood subgroup of lung

Correspondence to: Professor Ji-Feng Feng, Department of Chemotherapy, Nanjing Medical University Affiliated Cancer Hospital Cancer Institute of Jiangsu Province, Baiziting 42, Nanjing, Jiangsu 210009, P.R. China

E-mail: jifengfeng187@163.com

\section{${ }^{*}$ Contributed equally}

Key words: long non-coding RNA, EGFR-TKI, lung cancer, cis-regulation, trans-regulation adenocarcinomas (LACs). This subgroup is characterized by sensitivity to EGFR tyrosine kinase inhibitors (TKIs; e.g. gefitinib or erlotinib), and has high occurrence rates in females, non-smokers and Asians (1). However, a median therapy (10-14 months) with EGFR TKI may aggravate the disease progression in most patients in this subgroup. The mechanisms of EGFR-TKI resistance are multi-factorial and several of them have been reported, such as T790M mutation in exon 20 of EGFR, MET amplification and depletion of phosphatase and tensin homolog (PTEN) (2-4). However, nearly $30 \%$ of resistance mechanisms are unknown. Thus, elucidating the molecular mechanisms of EGFR-TKI resistance is essential for the identification of key biomarkers.

In the last two decades, non-coding RNAs (ncRNAs) have been gradually accepted as key factors in the process of epigenetic regulation rather than 'transcription noise' (5). As recently reported, ncRNAs take part in the pathogenesis of non-small cell lung cancer (NSCLC), providing new biological insights into this disease $(6,7)$. Long non-coding RNAs (lncRNAs), which are non-protein coding transcripts with length $>200$ nucleotides (nt), are involved in regulating the occurrence, invasion, metastasis and chemotherapeutic resistance of lung cancer $(6,8,9)$. IncRNAs also take part in the regulation of EGFR-TKI resistance. GAS5, one lncRNA, was found to be overexpressed in EGFR-TKI-sensitive cells compared with its expression in resistant cells, and was found to enhance gefitinib-induced cell death in innate EGFR-TKIresistant LAC cells with wild-type EGFR via downregulation of IGF-1R expression (10). These findings indicate that lncRNAs may be promising biomarkers as diagnostic and therapeutic targets in the resistance of EGFR-TKIs.

The present study presents the lncRNA expression profiles in 3 replicate gefitinib-sensitive $\mathrm{HCC} 827$ and gefitinib-resistant HCC827-8-1 cells in pairs by microarray. Then, 7 of the differentially expressed lncRNAs were validated by real-time quantitative PCR (RT-qPCR) in HCC827 and HCC8278-1 cells. We also predicted the functions of differentially expressed lncRNAs through their co-expressed protein-coding genes. 
Table I. The primer sequences used in the present study.

\begin{tabular}{lll}
\hline Target ID & \multicolumn{1}{c}{ Forward primer } & \multicolumn{1}{c}{ Reverse primer } \\
\hline FR165245 & GAGGGTTTGGCTGTTTGCTG & ACCCCCACTTAGAGACCAGAA \\
ENST00000464359 & GCAACAACCACTTGGCTCAG & GCAGAGGACACGAACTCACA \\
ENST00000602301 & GTTACCTCCTCATGCCGGAC & AAAAGGGTCAGTAAGCACCCG \\
NONHSAT107900 & GGCTGCATTTGTTTCTCGCA & CCCGCCCAGCTATAGTCAAG \\
NONHSAT082241 & TGCCAAAACTCACCAGCTACA & GGAGCGGTATGTGCTAGACC \\
ENST00000434951 & TGGGAGTGAATGTTCCGGTG & CAAGAGGAGCTGTTGTTTGTCC \\
NONHSAG031748 & GGATGTGCACGCATGAACTG & ACTCCAGCCAAGGTGGTTTT \\
$\beta$-actin & GATGAGATTGGCATGGCTTT & CACCTTCACCGTTCCAGTTT
\end{tabular}

\section{Materials and methods}

Cell culture. The human NSCLC H827 cell line harboring the EGFR exon 19 deletion (DelE746-A750) was obtained from the Shanghai Institutes for Biological Sciences, Chinese Academy of Cell Resource Center. We generated a gefitinib-resistant cell line by exposing HCC827 cells to increasing concentrations of gefitinib as described in our previous study (11). One individual clone HCC827-8-1 was isolated and independently confirmed to be resistant to gefitinib. HCC827-8-1 cells were 348 -fold more resistant to gefitinib than the parental HCC827 cells. The cells were cultured in RPMI-1640 medium supplemented with $10 \%$ fetal bovine serum (FBS) (Gibco, Carlsbad, CA, USA) at $37^{\circ} \mathrm{C}$, in a $5 \% \mathrm{CO}_{2}$ and humidified atmosphere.

RNA extraction. HCC827 and HCC827-8-1 cells were seeded in 6-well plates $\left(1 \times 10^{5}\right.$ cells/well) for $72 \mathrm{~h}$ in 3 replicate wells, and then resuspended in $500 \mu 1$ lysis buffer. Total RNA was extracted from lysis buffer using the mirVana miRNA Isolation kit procedure (Applied Biosystem, Foster City, CA, USA), according to the manufacturer's specifications, and eluted with $100 \mathrm{ml}$ of nuclease-free water. The yield of RNA was quantified by the NanoDrop ND 2000 (Thermo Scientific, Waltham, MA, USA) and the RNA integrity was assessed using Agilent Bioanalyzer 2100 (Agilent Technologies, Santa Clara, CA, USA).

lncRNA and mRNA microarray expression profiling. Total RNA was transcribed to double-stranded cDNA, and then synthesized into cRNA and labeled with cyanine 3-CTP. The labeled cRNAs were hybridized onto the microarray. The Agilent human IncRNA array $(4 \times 180 \mathrm{~K})$ contains 32,776 human mRNAs and 78,243 human IncRNAs, which are derived from authoritative databases, including RefSeq, Ensemble, GenBank and the Broad Institute. After washing, the arrays were scanned by the Agilent Scanner G2505C (Agilent Technologies). Raw datum was extracted using Feature Extraction (version 10.7.1.1; Agilent Technologies). The microarray profiling was conducted in the laboratory of the OE Biotechnology Co. (Shanghai, China).

RT-qPCR validation of 7 differentially expressed lncRNAs. Total RNA was extracted from HCC827 and HCC827-8-1 cells using TRIzol reagent (Invitrogen, Carlsbad, CA, USA) according to the manufacturer's instructions. One microgram of total RNA was reverse transcribed in a final volume of $20 \mu \mathrm{l}$ using PrimerScript RT Master Mix (Takara, Dalian, China). The reverse transcription reaction was carried out under the following conditions: $37^{\circ} \mathrm{C}$ for $15 \mathrm{~min} ; 85^{\circ} \mathrm{C}$ for $5 \mathrm{sec}$, and then hold on $4^{\circ} \mathrm{C}$. The RT-qPCR was performed using SYBR-Green PCR Mix (Roche, Mannheim, Germany) on the ABI 7900 system (Applied Biosystems) according to the manufacturer's instructions. The primer sequences are listed in Table I. $\beta$-actin was used as an internal control to normalize the amount of total RNA in each sample. Each sample was run in triplicate for analysis. At the end of the PCR cycles, melting curve analysis was performed to validate the specific generation of the expected PCR product. The expression levels of IncRNAs were normalized to internal control gene $\beta$-actin and were calculated using the $2^{-\Delta \Delta \mathrm{Ct}}$ method (12).

Differential expression level of $m R N A$ s and lncRNAs from the microarray. After quantile normalization, raw signals from the microarray were $\log 2$ transformed. Differential expression of an mRNA or IncRNA was defined by the absolute value of fold-change (FC) $>2$ (gefitinib-sensitive HCC827 cells=1) and P-value $<0.05$ (Student's t-test), with the 3 parallel samples in the HCC827 or HCC827-8-1 group having detectable signals compared with the background. The differentially expressed mRNAs were submitted to the NCBI (gene_go_information) and KEGG database analyzed by Python program to be classified into different Gene Ontology (GO) and Kyoto Encyclopedia of Genes and Genomes (KEGG) annotation groups.

Co-expression of lncRNAs with mRNAs and functional prediction. Most of the lncRNAs in the current databases have not yet been functionally annotated. Thus, the prediction of their functions is based on the functional annotations of their co-expressed mRNAs. This method was originally described by Guttman et al (13). In brief, first, for every dysregulated lncRNA, Pearson correlation coefficient (PCC) of its expression with that of each dysregulated mRNA was calculated to find its co-expressed mRNAs, with PCC $>0.7$ or $\leq 0.7$ and P-value of PCC $<0.05$ being statistically significant. Then, a functional enrichment analysis of the co-expressed mRNAs was conducted using the hypergeometric cumulative distribution function, and the enriched GO/KEGG pathway annotations were assigned to the lncRNA as its predicted 
Table II. Top 20 upregulated and downregulated lncRNAs and mRNAs in the HCC827 cells compared with the gefitinib-resistant HCC827-8-1 cells.

\begin{tabular}{|c|c|c|c|c|c|c|c|}
\hline \multicolumn{2}{|c|}{ Upregulated lncRNAs } & \multicolumn{2}{|c|}{ Downregulated lncRNAs } & \multicolumn{2}{|c|}{ Upregulated mRNAs } & \multicolumn{2}{|c|}{ Downregulated mRNAs } \\
\hline $\operatorname{lncRNAs}$ & FC & lncRNAs & FC & mRNAs & $\mathrm{FC}$ & mRNAs & $\mathrm{FC}$ \\
\hline ENST00000464359 & 136.11 & ENST00000602301 & 81.46 & FOXP2 & 74.25 & MCOLN2 & 31.03 \\
\hline NONHSAT130274 & 107.33 & NONHSAG002294 & 68.92 & PYDC2 & 29.44 & RNF183 & 27.43 \\
\hline NONHSAT122833 & 47.29 & ENST00000542980 & 33.87 & CHI3L1 & 26.90 & THNSL2 & 27.18 \\
\hline NONHSAT122828 & 38.98 & NONHSAT122257 & 23.86 & SFTPB & 19.99 & PGBD5 & 17.46 \\
\hline NONHSAG048587 & 30.11 & NONHSAG032896 & 22.43 & GJA1 & 16.79 & FAM26F & 15.87 \\
\hline uc. $225+$ & 27.11 & NONHSAT082241 & 22.04 & CTGF & 14.14 & ZNF655 & 15.23 \\
\hline NONHSAT122829 & 22.16 & NONHSAT060965 & 20.40 & CALB1 & 13.04 & IGFBP3 & 15.12 \\
\hline NONHSAT122825 & 19.67 & NONHSAT101176 & 19.36 & ATP8A1 & 12.95 & ZNF560 & 13.81 \\
\hline NONHSAT127642 & 14.70 & NONHSAT118619 & 15.69 & WNT9A & 10.87 & RPS6KA6 & 13.41 \\
\hline NONHSAT093968 & 14.13 & ENST00000423737 & 15.53 & SPTLC3 & 10.79 & FRMD3 & 13.17 \\
\hline NONHSAT060927 & 13.25 & ENST00000424690 & 15.04 & SPOCK1 & 9.81 & BACE2 & 13.16 \\
\hline NONHSAT127638 & 12.60 & NONHSAT120476 & 14.96 & SPTLC3 & 9.48 & GPR133 & 12.70 \\
\hline FR173955 & 12.50 & NONHSAG046899 & 14.92 & SFTPB & 9.16 & CYP4F12 & 12.26 \\
\hline NONHSAG050711 & 12.28 & NONHSAT118621 & 14.67 & BMP4 & 8.77 & PTGER2 & 12.23 \\
\hline XR_254376.1 & 11.98 & NONHSAT101177 & 13.85 & AQP3 & 8.62 & CKMT1A & 11.58 \\
\hline NONHSAT107900 & 11.85 & NONHSAT060785 & 12.80 & EGR1 & 8.27 & FAM129A & 11.11 \\
\hline NONHSAT103972 & 11.56 & NONHSAT082244 & 12.59 & TOX2 & 8.20 & C17orf104 & 10.12 \\
\hline uc.222+ & 10.73 & NONHSAT053086 & 12.33 & GMPR & 7.92 & ICAM2 & 9.63 \\
\hline TCONS_00012260 & 10.37 & NONHSAT122256 & 11.48 & ADORA1 & 7.90 & MUC22 & 9.61 \\
\hline FR 165245 & 9.81 & NONHSAT004232 & 11.24 & CEACAM6 & 7.81 & LOC643988 & 9.53 \\
\hline
\end{tabular}

FC, absolute fold-change (HCC827 cells set as 1).

functions. The threshold of statistical significance is set as a $\mathrm{P}$-value $<0.05$ and false discovery rate $(\mathrm{FDR})<0.05$ [under the control of the Benjamini and Hochberg procedure (14)].

In addition, on the basis of co-expression, we further explored how these dysregulated lncRNAs may exert their functions through cis-and/or trans-regulating protein-coding genes. We defined cis-regulated genes as protein-coding genes co-expressed with one dysregulated lncRNA and within $300 \mathrm{~kb}$ in genomic distance in the same allele.

We potentially defined trans-regulated protein-coding genes as co-expressed and beyond $300 \mathrm{~kb}$ in genomic distance from, or, on the other allele of, differentially expressed lncRNAs. In other words, for any protein-coding gene co-expressed with an IncRNA, if it did not fit the criteria of cis-regulated, it was categorized as potentially trans-regulated.

According to Guttman et al $(13,15)$, specific lncRNAs participating in certain biological pathways are transcriptionally regulated by key transcription factors (TFs) that regulate these pathways. Thus, to categorize IncRNAs that possibly have trans-regulating functions, we compared the mRNAs that co-expressed with these IncRNAs with the mRNAs that are regulatory targets of certain TFs. If the intersection of these 2 groups is large enough $(\mathrm{P}<0.05$; calculated by hypergeometric cumulative distribution function and FDR $<0.05$, under the control of the Benjamini and Hochberg procedure), then, we predict that these lncRNAs possibly participate in pathways regulated by these TFs. The lncRNA-TF network was constructed using hypergeometric cumulative distribution function with the help of Perl. The graph of the lncRNA-TF network was drawn with the help of Cytoscape 3.01.

\section{Results}

General expression profiles of the differentially expressed lncRNAs and mRNAs. We found that 1,476 lncRNA transcripts were differentially expressed between the HCC827-8-1 and paired HCC 827 cells, with 703 being upregulated and 773 being downregulated. Among the dysregulated lncRNA transcripts, ENST00000464359 (probe CUST_89973_PI429545380) was the most upregulated, with an FC of 136.11, whereas ENST00000602301 (probe CUST_1064_PI429545384) was the most downregulated, FC being 81.46. According to the absolute value of FC, the dysregulated lncRNA transcripts were stratified into 4 groups: 2 transcripts with FCs $>100$, 38 transcripts between 10 and 100, 117 transcripts between 5 and 10 and 1,319 transcripts between 2 and 5 .

Using the same criteria of IncRNAs, we found that 1,026 mRNA transcripts were dysregulated, with 516 being upregulated and 510 being downregulated. The most upregulated and downregulated mRNA transcripts were FOXP2 (A_24_P16559) and MCOLN2 (A_23_P23639), with FCs of 74.25 and 31.03 , respectively. Table II lists the top 20 upregulated and downregulated lncRNAs and mRNAs from our microarray. 


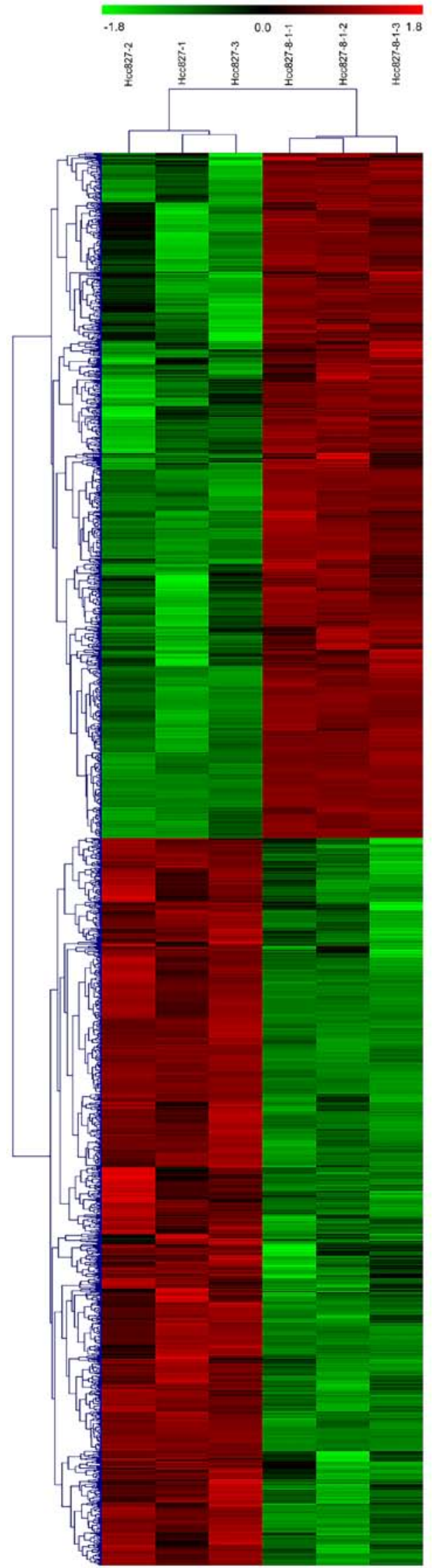

Figure 1. Heat map showing differentially expressed lncRNAs in the HCC827 cells compared with the gefitinib-resistant HCC827-8-1 cells. Each row represents one lncRNA, and each column represents one cell line sample. The relative lncRNA expression is depicted according to the color scale. Red indicates upregulation; green indicates downregulation; 1.8, 0 and -1.8 are fold-changes in the corresponding spectrum. The differentially expressed lncRNAs clearly self-segregated into HCC827 and HCC827-8-1 cell clusters
In the GO pathway analysis, the most common pathways that the dysregulated mRNAs were involved in included chondroitin sulfate metabolic process (GO, biological processes), extracellular space (GO, cellular components) and receptor binding (GO, molecular functions). The most common KEGG pathways involved were graft-versus-host disease, type I diabetes mellitus and viral myocarditis.

In unsupervised hierarchical clustering analysis, the differentially expressed lncRNAs were used to generate a heat map; and they clearly self-segregated into HCC827 and HCC827-8-1 clusters, as shown in Fig. 1.

$R T-q P C R$ validation. To validate the results of the microarray, we chose a total of 7 differentially expressed lncRNA transcripts for RT-qPCR. They could be divided into 2 groups: the first group, including FR165245, which was randomly chosen. In the other group, NONHSAT107900 and NONHSAT082241 were chosen since they were predicted to have $c i s$-regulating potential. ENST00000434951 and ONHSAG031748 were chosen since they were predicted to have trans-regulating potential and involved in constituting the top 100 lncRNA-TF pairs with the most credentiality. ENST00000464359 and ENST00000602301 were chosen for being the most upregulated and downregulated lncRNAs (Table II).

The RT-qPCR results were consistent with that of the microarray, in that all 7 lncRNA transcripts were differentially expressed with the same trend (upregulated or downregulated) and reached statistical significance $(\mathrm{P}<0.05$ for each lncRNA; Student's t-test) as shown in Fig. 2.

lncRNA and mRNA co-expression profiles and lncRNA function prediction. Hundreds of IncRNAs were co-expressed with thousands of mRNAs. For example, ENST00000412387 (CUST_86592_PI429545380) was co-expressed with 3,487 mRNA transcripts and NONHSAT060786 (CUST_61584_PI429545406) with 4,642 mRNA transcripts.

The functions of the differentially expressed lncRNAs were predicted by the GO and KEGG pathway annotations of their co-expressed mRNAs. The lncRNAs were clustered into hundreds of GO and KEGG pathway annotations. Certain pathways are known to be involved in the mechanism of gefitinib resistance, including focal adhesion, cell cycle, cell proliferation and apoptosis (16-18). For example, 186 lncRNAs were clustered into apoptosis and 79 in the focal adhesion. As expected, one lncRNA can participate in more than one GO/KEGG pathway involved in the mechanism of gefitinib resistance. For example, ENST00000412387 (CUST_86592_PI429545380) was predicted to have functions in apoptosis, cell cycle, focal adhesion and pathways in cancer.

From the matrix of the lncRNAs and their corresponding KEGG pathway annotations of co-expressed protein-coding genes, we counted and summarized the top 200 annotations with the most credentiality (the lowest P-values). The most frequently predicted functions of the differentially expressed lncRNAs were metabolic pathways, glyoxylate and dicarboxylate metabolism and N-glycan biosynthesis as shown in Fig. 3.

cis-regulation of lncRNAs. A total of 149 lncRNA transcripts with their predicted cis-regulated protein-coding genes were 


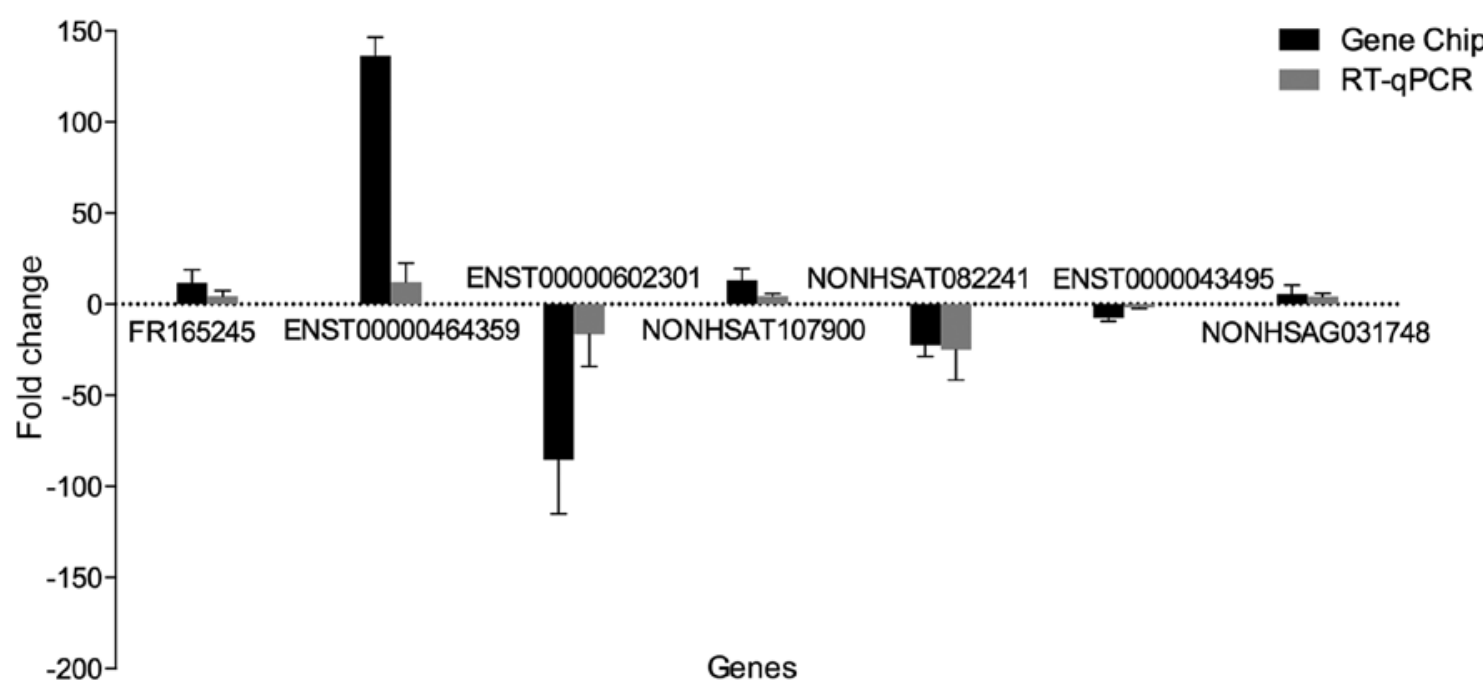

Figure 2. Real-time quantitative PCR results of the 7 chosen lncRNAs which validate those of the microarray ( $\mathrm{P}<0.05$; Student's t-test). One randomly chosen lncRNA. Six lncRNAs with potential biological significance elaborated in the present study or with the highest fold-change. The heights of the columns in the chart represent the mean fold-change in expression for each of these lncRNAs. Bars represent SEM. Fold-change was positive when the expression was upregulated (HCC827-8-1/HCC827 cells) and negative when downregulated.

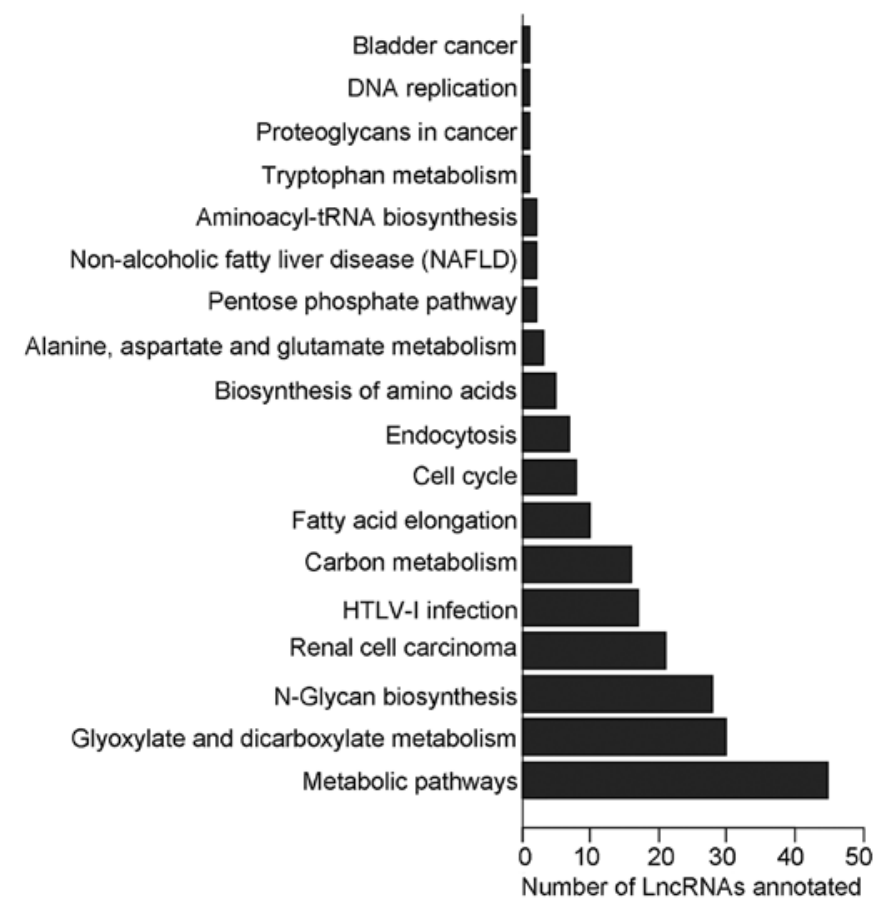

Figure 3. Top 200 hits of KEGG pathway annotations of the differentially expressed lncRNAs. The $\mathrm{x}$-axis shows the number of lncRNAs annotated and the $y$-axis shows the GO annotations.

found through accurate genomic mapping, using the above mentioned criteria. Table III lists all the lncRNA transcripts and their potentially cis-regulated mRNA transcripts. For instance, lncRNA NONHSAG010348 was predicted to cis-regulate five mRNAs (corresponding to five mRNA transcripts): COPS7A, TPI1, EMG1, USP5 and LRRC23.

Trans-regulation of the lncRNAs. Using the threshold of $\mathrm{P}<0.05$ and FDR $<0.01,3,429$ lncRNA-TF pairs were found, corresponding to 48 TFs. Then, we generated a core network using the top 100 lncRNA-TF pairs with the most credentiality (lowest P-values and FDRs), as shown by Fig. 4 and are listed in Table IV. We can see that most of these potential trans-regulatory lncRNAs participate in pathways regulated by four TFs: E2F transcription factor 1 (E2F1), E2F transcription factor 4 (E2F4), upstream transcription factor 1 (USF1) and transcription factor AP-2 $\gamma$ (TFAP2C). In the core network of lncRNA-TF pairs, E2F1 participates in 31 of the 100 pairs, E2F4 in 10 pairs, USF1 in 17 pairs and TFAP2C in 30 pairs.

\section{Discussion}

We assessed genome-wide lncRNA expression patterns in gefitinib-resistant human NSCLC HCC827-8-1 cells, compared with their parental HCC827 cells by microarray and explored their possible functions by analyzing their co-expressed protein-coding mRNAs. HCC827-8-1 is an individual clone isolated from resistant HCC827 cells, which is 348 -fold more resistant to gefitinib than HCC827 cells with high stability (11). We used HCC827-8-1 cells to study the EGFR-TKI resistance mechanisms and to obtain more accurate results.

The microarray showed that 1,476 1ncRNA and 1,026 mRNA transcripts were dysregulated. Then we chose and validated 7 of the differentially expressed 1 ncRNAs by RT-qPCR. Hundreds of lncRNAs were co-expressed with thousands of mRNAs, and some of the lncRNAs may be active in the mechanism of gefitinib resistance through $c i s$-regulation and/or in trans-regulation of these mRNAs.

We predicted the roles of IncRNAs through the co-expressed mRNAs. The most widely used technique for function prediction is the 'guilt by association' method, which aims to interrogate the co-expressed protein-coding genes and relevant bio-pathways (13). Among the hundreds of pathways as-predicted, some are key pathways in the mechanisms of gefitinib resistance, such as focal adhesion, cell proliferation, cell cycle and apoptosis (16-18). We compared the differentially expressed mRNAs with other studies, and the high 
Table III. Dysregulated lncRNA transcripts and their potentially cis-regulated mRNA transcripts.

\begin{tabular}{|c|c|c|c|c|}
\hline lncRNA & mRNA & PCC & P-value ${ }^{a}$ & Chrom \\
\hline ENST00000423737 & LOC284930 & 0.990763962 & 0.000128 & 22 \\
\hline ENST00000425104 & SLC35F3 & -0.871875729 & 0.023572 & 1 \\
\hline ENST00000431813 & DAPK1 & 0.944254301 & 0.004575 & 9 \\
\hline ENST00000438810 & LOC284930 & 0.988689739 & 0.000191 & 22 \\
\hline ENST00000499202 & $\mathrm{CD} 27$ & -0.927815863 & 0.007628 & 12 \\
\hline ENST00000499202 & COPS7A & -0.915741752 & 0.010350 & 12 \\
\hline ENST00000499202 & GAPDH & -0.820431489 & 0.045472 & 12 \\
\hline ENST00000499202 & LTBR & -0.958751121 & 0.002517 & 12 \\
\hline ENST00000499202 & NCAPD2 & -0.926349955 & 0.007937 & 12 \\
\hline ENST00000499202 & PLEKHG6 & 0.886477026 & 0.018600 & 12 \\
\hline ENST00000508616 & OR5H15 & -0.914857473 & 0.010565 & 3 \\
\hline ENST00000510682 & COMMD10 & 0.931176055 & 0.006942 & 5 \\
\hline ENST00000558536 & C15orf48 & 0.948581359 & 0.003898 & 15 \\
\hline ENST00000563635 & LMO7 & 0.962112937 & 0.002126 & 13 \\
\hline ENST00000567305 & RHBDD1 & -0.860850737 & 0.027697 & 2 \\
\hline ENST00000605692 & ATP6V1F & 0.869839077 & 0.024310 & 7 \\
\hline ENST00000605692 & CCDC136 & 0.829361383 & 0.041192 & 7 \\
\hline ENST00000605692 & FAM71F1 & 0.889362332 & 0.017684 & 7 \\
\hline ENST00000605692 & FAM71F2 & 0.919989247 & 0.009346 & 7 \\
\hline ENST00000605692 & HILPDA & -0.983130671 & 0.000424 & 7 \\
\hline ENST00000605692 & IRF5 & 0.95923013 & 0.002459 & 7 \\
\hline ENST00000605692 & LOC100130705 & 0.954248076 & 0.003092 & 7 \\
\hline ENST00000606008 & ZNF35 & 0.890496793 & 0.017330 & 3 \\
\hline ENST00000606008 & ZNF501 & 0.847741084 & 0.033009 & 3 \\
\hline FR009982 & LOC100131490 & -0.86144213 & 0.027467 & 12 \\
\hline FR070335 & ADAMTS15 & 0.915157825 & 0.010492 & 11 \\
\hline FR070335 & ST14 & -0.911123761 & 0.011497 & 11 \\
\hline FR078172 & SENP3 & 0.891854464 & 0.016911 & 17 \\
\hline FR078172 & WRAP53 & 0.851342123 & 0.031506 & 17 \\
\hline FR085258 & PIM3 & 0.89707096 & 0.015346 & 22 \\
\hline FR148984 & COQ10A & -0.918928534 & 0.009592 & 12 \\
\hline FR148984 & ESYT1 & -0.859157792 & 0.028358 & 12 \\
\hline FR148984 & GDF11 & -0.861243067 & 0.027544 & 12 \\
\hline FR148984 & MYL6 & -0.939508853 & 0.005378 & 12 \\
\hline FR148984 & MYL6B & -0.818664102 & 0.046343 & 12 \\
\hline FR148984 & OBFC2B & 0.949121375 & 0.003817 & 12 \\
\hline FR148984 & ORMDL2 & -0.938671908 & 0.005526 & 12 \\
\hline FR148984 & RAB5B & -0.92713364 & 0.007771 & 12 \\
\hline FR171954 & SEMA4B & -0.901277827 & 0.014138 & 15 \\
\hline FR230846 & SERF1B & 0.949144988 & 0.003814 & 5 \\
\hline FR314507 & KCNK6 & -0.914052441 & 0.010763 & 19 \\
\hline FR314507 & SIPA1L3 & 0.900771256 & 0.014281 & 19 \\
\hline FR314507 & SPRED3 & 0.885802839 & 0.018817 & 19 \\
\hline FR331033 & EFNB 1 & 0.967665213 & 0.001551 & $X$ \\
\hline NONHSAG002294 & NTNG1 & 0.835837077 & 0.038212 & 1 \\
\hline NONHSAG004747 & LGALS8 & 0.941744836 & 0.004992 & 1 \\
\hline NONHSAG006325 & PPIF & -0.832471447 & 0.039748 & 10 \\
\hline NONHSAG006325 & ZMIZ1 & 0.944693182 & 0.004504 & 10 \\
\hline NONHSAG010348 & COPS7A & 0.984517242 & 0.000358 & 12 \\
\hline NONHSAG010348 & EMG1 & 0.931371288 & 0.006903 & 12 \\
\hline NONHSAG010348 & LRRC23 & 0.900711998 & 0.014298 & 12 \\
\hline NONHSAG010348 & TPI1 & 0.953578734 & 0.003182 & 12 \\
\hline
\end{tabular}


Table III. Continued.

\begin{tabular}{|c|c|c|c|c|}
\hline lncRNA & mRNA & PCC & P-value & Chrom \\
\hline NONHSAG010348 & USP5 & 0.918972659 & 0.009582 & 12 \\
\hline NONHSAG010749 & ARNTL2 & 0.917332369 & 0.009968 & 12 \\
\hline NONHSAG025545 & FXYD5 & 0.924498207 & 0.008336 & 19 \\
\hline NONHSAG025545 & FXYD7 & -0.927092642 & 0.007779 & 19 \\
\hline NONHSAG025545 & ZNF181 & 0.893862023 & 0.016300 & 19 \\
\hline NONHSAG025545 & ZNF30 & 0.93136471 & 0.006905 & 19 \\
\hline NONHSAG025545 & ZNF302 & 0.830684654 & 0.040575 & 19 \\
\hline NONHSAG028996 & IL1RN & 0.956644173 & 0.002779 & 2 \\
\hline NONHSAG034063 & SERHL2 & 0.905613504 & 0.012943 & 22 \\
\hline NONHSAG035134 & HEMK1 & -0.964351703 & 0.001884 & 3 \\
\hline NONHSAG035134 & MAPKAPK3 & 0.817490481 & 0.046925 & 3 \\
\hline NONHSAG037825 & DCAF4L1 & -0.886889957 & 0.018467 & 4 \\
\hline NONHSAG039533 & CYP4V2 & 0.967426574 & 0.001574 & 4 \\
\hline NONHSAG039533 & FAM149A & 0.986343279 & 0.000278 & 4 \\
\hline NONHSAG041216 & MAN2A1 & -0.863109335 & 0.026826 & 5 \\
\hline NONHSAG042317 & C5orf 25 & 0.898935246 & 0.014805 & 5 \\
\hline NONHSAG042611 & FOXQ1 & -0.908075468 & 0.012287 & 6 \\
\hline NONHSAG047857 & C7orf42 & 0.950970866 & 0.003547 & 7 \\
\hline NONHSAG048587 & MDFIC & 0.942137396 & 0.004925 & 7 \\
\hline NONHSAG049128 & GIMAP4 & -0.859295231 & 0.028304 & 7 \\
\hline NONHSAG049128 & GIMAP8 & -0.900385381 & 0.014390 & 7 \\
\hline NONHSAG049128 & REPIN1 & -0.912314796 & 0.011196 & 7 \\
\hline NONHSAG052187 & DCAF10 & -0.886559031 & 0.018573 & 9 \\
\hline NONHSAG052187 & RG9MTD3 & 0.974819506 & 0.000943 & 9 \\
\hline NONHSAG052737 & DAPK1 & 0.991651051 & 0.000104 & 9 \\
\hline NONHSAG053520 & COQ4 & 0.816256551 & 0.047541 & 9 \\
\hline NONHSAT002986 & C1orf190 & 0.944107317 & 0.004599 & 1 \\
\hline NONHSAT002986 & LOC100133124 & -0.923578795 & 0.008537 & 1 \\
\hline NONHSAT002986 & NSUN4 & -0.856846302 & 0.029273 & 1 \\
\hline NONHSAT002986 & RAD54L & -0.864794791 & 0.026185 & 1 \\
\hline NONHSAT004254 & CYR61 & 0.990548945 & 0.000134 & 1 \\
\hline NONHSAT004989 & GPSM2 & 0.887936322 & 0.018134 & 1 \\
\hline NONHSAT005081 & CSF1 & 0.870913412 & 0.023920 & 1 \\
\hline NONHSAT005942 & ANKRD34A & 0.91139166 & 0.011429 & 1 \\
\hline NONHSAT005942 & LIX1L & 0.969652404 & 0.001367 & 1 \\
\hline NONHSAT005942 & PDZK1 & 0.982557048 & 0.000454 & 1 \\
\hline NONHSAT005942 & RBM8A & 0.86427635 & 0.026381 & 1 \\
\hline NONHSAT005942 & RNF115 & -0.849741206 & 0.032170 & 1 \\
\hline NONHSAT006288 & C1orf51 & 0.888664251 & 0.017903 & 1 \\
\hline NONHSAT006288 & CA14 & 0.895183669 & 0.015904 & 1 \\
\hline NONHSAT006288 & PRPF3 & 0.812873365 & 0.049248 & 1 \\
\hline NONHSAT006799 & LAMTOR2 & 0.877579069 & 0.021563 & 1 \\
\hline NONHSAT006799 & LMNA & -0.940089303 & 0.005276 & 1 \\
\hline NONHSAT006799 & PMF1-BGLAP & -0.865484674 & 0.025925 & 1 \\
\hline NONHSAT006799 & RXFP4 & -0.816428591 & 0.047455 & 1 \\
\hline NONHSAT006799 & SEMA4A & -0.865518945 & 0.025912 & 1 \\
\hline NONHSAT006799 & SLC25A44 & -0.92529896 & 0.008162 & 1 \\
\hline NONHSAT006799 & TMEM79 & 0.864331726 & 0.026360 & 1 \\
\hline NONHSAT007139 & NDUFS2 & -0.811560982 & 0.049918 & 1 \\
\hline NONHSAT007139 & NIT1 & 0.850528627 & 0.031843 & 1 \\
\hline NONHSAT007139 & TOMM40L & 0.981849759 & 0.000491 & 1 \\
\hline NONHSAT007139 & UFC1 & 0.91161181 & 0.011373 & 1 \\
\hline
\end{tabular}


Table III. Continued.

\begin{tabular}{|c|c|c|c|c|}
\hline lncRNA & mRNA & PCC & P-value ${ }^{a}$ & Chrom \\
\hline NONHSAT007139 & USP21 & -0.908563094 & 0.012159 & 1 \\
\hline NONHSAT010406 & LGALS8 & 0.920135293 & 0.009313 & 1 \\
\hline NONHSAT010413 & LGALS8 & 0.934268515 & 0.006339 & 1 \\
\hline NONHSAT010414 & LGALS8 & 0.94625791 & 0.004255 & 1 \\
\hline NONHSAT011117 & AKR1E2 & 0.887463422 & 0.018284 & 10 \\
\hline NONHSAT016347 & DUSP5 & 0.923683122 & 0.008514 & 10 \\
\hline NONHSAT016347 & MXI1 & -0.921167433 & 0.009077 & 10 \\
\hline NONHSAT016347 & RBM20 & 0.816015258 & 0.047662 & 10 \\
\hline NONHSAT018220 & NUCB2 & 0.968114991 & 0.001509 & 11 \\
\hline NONHSAT018821 & LDLRAD3 & 0.830563596 & 0.040631 & 11 \\
\hline NONHSAT021952 & C11orf20 & -0.939345932 & 0.005407 & 11 \\
\hline NONHSAT021952 & COX8A & -0.849229323 & 0.032384 & 11 \\
\hline NONHSAT021952 & FERMT3 & 0.908490858 & 0.012178 & 11 \\
\hline NONHSAT021952 & FKBP2 & 0.880614632 & 0.020529 & 11 \\
\hline NONHSAT021952 & NAA40 & 0.950492833 & 0.003616 & 11 \\
\hline NONHSAT021952 & OTUB1 & 0.96848133 & 0.001474 & 11 \\
\hline NONHSAT021952 & PLCB3 & -0.927522493 & 0.007689 & 11 \\
\hline NONHSAT021952 & PRDX5 & 0.873645855 & 0.022939 & 11 \\
\hline NONHSAT021952 & STIP1 & 0.97759167 & 0.000748 & 11 \\
\hline NONHSAT021952 & VEGFB & -0.98828861 & 0.000205 & 11 \\
\hline NONHSAT023878 & BIRC2 & 0.879025568 & 0.021067 & 11 \\
\hline NONHSAT023878 & C11orf70 & 0.932341202 & 0.006712 & 11 \\
\hline NONHSAT026185 & COPS7A & 0.988490936 & 0.000198 & 12 \\
\hline NONHSAT026185 & EMG1 & 0.942136719 & 0.004925 & 12 \\
\hline NONHSAT026185 & LRRC23 & 0.928925218 & 0.007398 & 12 \\
\hline NONHSAT026185 & PTPN6 & 0.831260781 & 0.040307 & 12 \\
\hline NONHSAT026185 & TPI1 & 0.964345232 & 0.001884 & 12 \\
\hline NONHSAT026185 & USP5 & 0.935201329 & 0.006162 & 12 \\
\hline NONHSAT028274 & SCN8A & -0.926130113 & 0.007984 & 12 \\
\hline NONHSAT028274 & SLC4A8 & 0.88116699 & 0.020343 & 12 \\
\hline NONHSAT028356 & C12orf44 & 0.989758866 & 0.000157 & 12 \\
\hline NONHSAT030840 & LOC100129447 & 0.824754125 & 0.043376 & 12 \\
\hline NONHSAT031072 & HSPB8 & 0.955579328 & 0.002916 & 12 \\
\hline NONHSAT034980 & ITGBL1 & 0.960755647 & 0.002280 & 13 \\
\hline NONHSAT035015 & ERCC5 & 0.814310544 & 0.048520 & 13 \\
\hline NONHSAT037446 & ARG2 & 0.928999759 & 0.007383 & 14 \\
\hline NONHSAT037446 & PLEKHH1 & 0.983562296 & 0.000403 & 14 \\
\hline NONHSAT037452 & ARG2 & 0.937491109 & 0.005739 & 14 \\
\hline NONHSAT037452 & PLEKHH1 & 0.974920148 & 0.000936 & 14 \\
\hline NONHSAT051795 & NME4 & 0.885330103 & 0.018970 & 16 \\
\hline NONHSAT051795 & RAB40C & 0.889230112 & 0.017725 & 16 \\
\hline NONHSAT051795 & WFIKKN1 & 0.837341388 & 0.037535 & 16 \\
\hline NONHSAT054629 & LOC100130580 & 0.875832347 & 0.022169 & 17 \\
\hline NONHSAT054629 & PDK2 & 0.895328542 & 0.015861 & 17 \\
\hline NONHSAT054629 & RSAD1 & -0.87520844 & 0.022388 & 17 \\
\hline NONHSAT054629 & SPATA20 & 0.868769216 & 0.024702 & 17 \\
\hline NONHSAT054629 & TMEM92 & 0.988506098 & 0.000197 & 17 \\
\hline NONHSAT056331 & CCDC40 & 0.938792121 & 0.005505 & 17 \\
\hline NONHSAT056331 & SLC26A11 & 0.868405134 & 0.024836 & 17 \\
\hline NONHSAT060927 & ANGPTL4 & 0.946117654 & 0.004277 & 19 \\
\hline NONHSAT060927 & HNRNPM & 0.913205652 & 0.010973 & 19 \\
\hline NONHSAT060927 & 2-Mar & 0.8868303 & 0.018486 & 19 \\
\hline
\end{tabular}


Table III. Continued.

\begin{tabular}{|c|c|c|c|c|}
\hline lncRNA & mRNA & PCC & P-value ${ }^{a}$ & Chrom \\
\hline NONHSAT060927 & OR2Z1 & 0.917317857 & 0.009972 & 19 \\
\hline NONHSAT060927 & RAB11B & 0.95392537 & 0.003135 & 19 \\
\hline NONHSAT066483 & CEACAM3 & 0.990570871 & 0.000133 & 19 \\
\hline NONHSAT066483 & CEACAM6 & 0.974843312 & 0.000941 & 19 \\
\hline NONHSAT074144 & WBP11 & -0.924683038 & 0.008295 & 2 \\
\hline NONHSAT075349 & PHOSPHO2 & 0.918978696 & 0.009581 & 2 \\
\hline NONHSAT077950 & FARP2 & 0.842995975 & 0.035040 & 2 \\
\hline NONHSAT077950 & ING5 & 0.919706694 & 0.009412 & 2 \\
\hline NONHSAT077950 & LOC100129675 & 0.945435006 & 0.004385 & 2 \\
\hline NONHSAT082241 & BACE2 & 0.97176878 & 0.001184 & 21 \\
\hline NONHSAT083016 & COL6A2 & 0.84242078 & 0.035290 & 21 \\
\hline NONHSAT086623 & LOC100652769 & 0.947578927 & 0.004050 & 22 \\
\hline NONHSAT086623 & NOL12 & 0.831885348 & 0.040018 & 22 \\
\hline NONHSAT086623 & SH3BP1 & 0.964024253 & 0.001918 & 22 \\
\hline NONHSAT086623 & TRIOBP & 0.956519314 & 0.002795 & 22 \\
\hline NONHSAT086830 & FAM83F & 0.935060728 & 0.006189 & 22 \\
\hline NONHSAT089680 & KLHDC8B & 0.968550457 & 0.001468 & 3 \\
\hline NONHSAT089680 & TCTA & 0.820457886 & 0.045459 & 3 \\
\hline NONHSAT090112 & PXK & 0.98306533 & 0.000428 & 3 \\
\hline NONHSAT092654 & TM4SF4 & -0.900748794 & 0.014287 & 3 \\
\hline NONHSAT092847 & GMPS & 0.890300599 & 0.017391 & 3 \\
\hline NONHSAT093875 & HRG & -0.937122594 & 0.005806 & 3 \\
\hline NONHSAT093875 & ST6GAL1 & 0.987402781 & 0.000237 & 3 \\
\hline NONHSAT093968 & CCDC50 & 0.884575249 & 0.019215 & 3 \\
\hline NONHSAT093968 & PYDC2 & 0.963323686 & 0.001993 & 3 \\
\hline NONHSAT094657 & FGFRL1 & -0.957980604 & 0.002611 & 4 \\
\hline NONHSAT094657 & SPON2 & 0.925043387 & 0.008217 & 4 \\
\hline NONHSAT095682 & FLJ39653 & 0.924889987 & 0.008250 & 4 \\
\hline NONHSAT096163 & LIMCH1 & 0.972549746 & 0.001120 & 4 \\
\hline NONHSAT096163 & UCHL1 & 0.918539886 & 0.009683 & 4 \\
\hline NONHSAT096168 & DCAF4L1 & -0.868194518 & 0.024914 & 4 \\
\hline NONHSAT096168 & LIMCH1 & 0.991159228 & 0.000117 & 4 \\
\hline NONHSAT099638 & CYP4V2 & 0.942816522 & 0.004811 & 4 \\
\hline NONHSAT099638 & FAM149A & 0.986547438 & 0.000270 & 4 \\
\hline NONHSAT099643 & CYP4V2 & 0.969996619 & 0.001337 & 4 \\
\hline NONHSAT099643 & FAM149A & 0.920009458 & 0.009342 & 4 \\
\hline NONHSAT101145 & PTGER4 & 0.876795864 & 0.021834 & 5 \\
\hline NONHSAT105337 & C5orf25 & 0.946965914 & 0.004144 & 5 \\
\hline NONHSAT107900 & GMPR & 0.924577154 & 0.008318 & 6 \\
\hline NONHSAT107917 & CAP2 & 0.969062042 & 0.001421 & 6 \\
\hline NONHSAT107973 & KDM1B & 0.946171234 & 0.004268 & 6 \\
\hline NONHSAT113449 & C6orf57 & 0.849248329 & 0.032376 & 6 \\
\hline NONHSAT114226 & PRDM1 & 0.938342631 & 0.005585 & 6 \\
\hline NONHSAT119003 & C7orf26 & 0.987782187 & 0.000223 & 7 \\
\hline NONHSAT119003 & RAC1 & 0.935823609 & 0.006046 & 7 \\
\hline NONHSAT119451 & DNAH11 & 0.985235314 & 0.000325 & 7 \\
\hline NONHSAT119452 & DNAH11 & 0.980786037 & 0.000550 & 7 \\
\hline NONHSAT119495 & KLHL7 & -0.917044909 & 0.010037 & 7 \\
\hline NONHSAT120737 & ССТ6А & 0.898610052 & 0.014899 & 7 \\
\hline NONHSAT120737 & GBAS & 0.948686683 & 0.003882 & 7 \\
\hline NONHSAT120737 & MRPS17 & 0.811824769 & 0.049783 & 7 \\
\hline NONHSAT120737 & SUMF2 & 0.948263714 & 0.003946 & 7 \\
\hline
\end{tabular}


Table III. Continued.

\begin{tabular}{|c|c|c|c|c|}
\hline $\operatorname{lncRNA}$ & mRNA & PCC & P-value ${ }^{a}$ & Chrom \\
\hline NONHSAT121617 & $\mathrm{ZP3}$ & 0.861523702 & 0.027436 & 7 \\
\hline NONHSAT122253 & CPSF4 & 0.908475946 & 0.012182 & 7 \\
\hline NONHSAT122253 & ZNF655 & 0.949103779 & 0.003820 & 7 \\
\hline NONHSAT122253 & ZNF789 & -0.94433753 & 0.004561 & 7 \\
\hline NONHSAT122254 & CPSF4 & 0.937348472 & 0.005765 & 7 \\
\hline NONHSAT122254 & ZNF655 & 0.949540597 & 0.003755 & 7 \\
\hline NONHSAT122254 & ZNF789 & -0.841096553 & 0.035869 & 7 \\
\hline NONHSAT122256 & CPSF4 & 0.94869328 & 0.003881 & 7 \\
\hline NONHSAT122256 & ZNF655 & 0.982379785 & 0.000463 & 7 \\
\hline NONHSAT122256 & ZNF789 & -0.891613102 & 0.016985 & 7 \\
\hline NONHSAT122257 & CPSF4 & 0.979295885 & 0.000639 & 7 \\
\hline NONHSAT122257 & ZNF789 & -0.90462309 & 0.013211 & 7 \\
\hline NONHSAT122826 & FOXP2 & 0.984524991 & 0.000357 & 7 \\
\hline NONHSAT122828 & FOXP2 & 0.963307259 & 0.001995 & 7 \\
\hline NONHSAT122828 & MDFIC & 0.957629177 & 0.002655 & 7 \\
\hline NONHSAT122829 & FOXP2 & 0.972231129 & 0.001146 & 7 \\
\hline NONHSAT122829 & MDFIC & 0.890381753 & 0.017366 & 7 \\
\hline NONHSAT122833 & MDFIC & 0.925397662 & 0.008141 & 7 \\
\hline NONHSAT122928 & CAPZA2 & 0.959462687 & 0.002432 & 7 \\
\hline NONHSAT122928 & ST7 & 0.98816268 & 0.000209 & 7 \\
\hline NONHSAT122929 & CAPZA2 & 0.953017879 & 0.003259 & 7 \\
\hline NONHSAT123242 & ATP6V1F & 0.936690382 & 0.005885 & 7 \\
\hline NONHSAT123242 & CCDC136 & 0.869648072 & 0.024380 & 7 \\
\hline NONHSAT123242 & HILPDA & -0.843372365 & 0.034877 & 7 \\
\hline NONHSAT123242 & LOC100130705 & 0.843594245 & 0.034781 & 7 \\
\hline NONHSAT125539 & LOC254896 & 0.873918742 & 0.022843 & 8 \\
\hline NONHSAT125539 & TNFRSF10C & 0.976171332 & 0.000845 & 8 \\
\hline NONHSAT127008 & ADHFE1 & 0.832589152 & 0.039694 & 8 \\
\hline NONHSAT127008 & RRS1 & 0.827125523 & 0.042245 & 8 \\
\hline NONHSAT129523 & LY6E & 0.868444037 & 0.024822 & 8 \\
\hline NONHSAT129523 & ZFP41 & 0.875838462 & 0.022167 & 8 \\
\hline NONHSAT129523 & ZNF696 & -0.885112169 & 0.019041 & 8 \\
\hline NONHSAT134920 & LCN2 & 0.960849658 & 0.002269 & 9 \\
\hline NONHSAT134920 & ODF2 & 0.86450331 & 0.026295 & 9 \\
\hline NONHSAT136475 & SMS & -0.976071345 & 0.000852 & $\mathrm{X}$ \\
\hline NONHSAT136568 & IL1RAPL1 & 0.976788703 & 0.000802 & $\mathrm{X}$ \\
\hline NONHSAT140499 & ATF7IP2 & 0.881735051 & 0.020153 & 16 \\
\hline NONHSAT140500 & ATF7IP2 & 0.913532878 & 0.010892 & 16 \\
\hline NONHSAT140506 & ATF7IP2 & 0.839342422 & 0.036643 & 16 \\
\hline NONHSAT140507 & ATF7IP2 & 0.835899063 & 0.038184 & 16 \\
\hline NONHSAT142843 & CCDC113 & -0.952964178 & 0.003267 & 16 \\
\hline NONHSAT142843 & NDRG4 & 0.983697804 & 0.000396 & 16 \\
\hline NONHSAT142849 & $\mathrm{CCDC} 113$ & -0.959233693 & 0.002459 & 16 \\
\hline NONHSAT145291 & TXNDC17 & 0.914040222 & 0.010766 & 17 \\
\hline NONHSAT145291 & XAF1 & 0.990386145 & 0.000138 & 17 \\
\hline NR_002834.1 & OBSCN & 0.855142567 & 0.029956 & 1 \\
\hline NR_002834.1 & RNF187 & -0.865681416 & 0.025851 & 1 \\
\hline NR_027459.2 & SYT14 & 0.871422894 & 0.023735 & 1 \\
\hline NR_027621.1 & GPR112 & -0.818615818 & 0.046367 & $\mathrm{X}$ \\
\hline NR_040072.1 & $\mathrm{CCDC} 113$ & -0.975161687 & 0.000918 & 16 \\
\hline NR_040072.1 & NDRG4 & 0.989689573 & 0.000159 & 16 \\
\hline NR_046396.1 & LOC100652883 & -0.816083992 & 0.047627 & 17 \\
\hline
\end{tabular}


Table III. Continued.

\begin{tabular}{|c|c|c|c|c|}
\hline lncRNA & mRNA & PCC & P-value ${ }^{a}$ & Chrom \\
\hline NR_046396.1 & SLC16A13 & -0.852523846 & 0.031020 & 17 \\
\hline NR_046396.1 & TXNDC17 & 0.93099277 & 0.006979 & 17 \\
\hline NR_046396.1 & XAF1 & 0.97916361 & 0.000647 & 17 \\
\hline NR_073032.1 & KCNK6 & -0.945478809 & 0.004378 & 19 \\
\hline NR_073032.1 & SIPA1L3 & 0.942774847 & 0.004818 & 19 \\
\hline NR_073032.1 & SPRED3 & 0.955022355 & 0.002989 & 19 \\
\hline NR_102308.1 & MYADM & 0.856205852 & 0.029529 & 19 \\
\hline NR_102308.1 & PRKCG & 0.950132007 & 0.003668 & 19 \\
\hline NR_102308.1 & TSEN34 & 0.863372316 & 0.026725 & 19 \\
\hline NR_103533.1 & RUNX2 & -0.823610352 & 0.043926 & 6 \\
\hline TCONS_00001307 & SRP9 & 0.881615264 & 0.020193 & 1 \\
\hline TCONS_00009873 & CCT5 & -0.874191191 & 0.022746 & 5 \\
\hline TCONS_00009873 & 6-Mar & 0.886701263 & 0.018528 & 5 \\
\hline TCONS_00012995 & ANLN & -0.859883705 & 0.028073 & 7 \\
\hline TCONS_00013626 & GIMAP4 & -0.815740282 & 0.047800 & 7 \\
\hline TCONS_00013626 & GIMAP8 & -0.857174368 & 0.029142 & 7 \\
\hline TCONS_00013626 & REPIN1 & -0.931532021 & 0.006871 & 7 \\
\hline TCONS_00026596 & CCDC165 & 0.877457033 & 0.021605 & 18 \\
\hline TCONS_00026596 & LOC284219 & 0.870249439 & 0.024161 & 18 \\
\hline TCONS_00027489 & CYP4F12 & 0.968533169 & 0.001470 & 19 \\
\hline TCONS_00027489 & CYP4F3 & 0.979154806 & 0.000647 & 19 \\
\hline TCONS_00027489 & CYP4F8 & 0.831544704 & 0.040176 & 19 \\
\hline TCONS_00027489 & TPM4 & 0.830964632 & 0.040445 & 19 \\
\hline TCONS_12_00015621 & PSMD1 & 0.869747246 & 0.024344 & 2 \\
\hline TCONS_12_00021700 & C4orf29 & 0.873207805 & 0.023095 & 4 \\
\hline
\end{tabular}

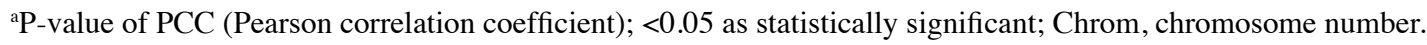

consistency strongly supported our predictions. For example, in the mRNA expression analysis using Agilent SurePrint G3 Human Gene Expression 8x60K array of a gefitinib-resistant lung cancer cell line (19), IGFBP3 was found to be expressed differentially, which is in accordance with our results. Additionally, the constitutive activation of EGFR tyrosine kinase receptor caused by mutation and/or amplification is closely correlated with the development, progression and poor prognosis of NSCLC, through the activation of various downstream signaling pathways. These pathways include the RAS/RAF/MAPK pathway that induces signals associated with proliferative activity, and the PI3K/AKT pathway that results in an anti-apoptotic effect (16). In the present study, the expression levels of AKT and RAS were higher in the HCC827-8-1 cells than that in the HCC827 cells.

cis-regulation is the intrinsic property of ncRNAs, since mRNAs are only effective in dissociation, transportation and translation (5). Moreover, the cis-regulation of nearby protein-coding genes is a common mechanism of lncRNAs (20). In the present study, we identified 149 lncRNA transcripts that were able to cis-regulate the nearby proteincoding genes. Although most of the 149 lncRNAs were uncharacterized, several of the lncRNA-mRNAs attracted our interest. IncRNAs that are related to dysregulated mRNAs known to be involved in EGFR-TKI resistance are valuable for further study. For example, one lncRNA is NONHSAT021952 (CUST_7952_PI429545402), which probably cis-regulates VEGF-B mRNA. As reported, VEGF-B plays important roles in promoting cancer metastasis through the remodeling of microvasculature of NSCLC, which is related to EGFR-TKI resistance (21). NONHSAT021952 (chr11: 6398830663990592+) has no annotated functions and lies near VEGF-B (chr11: 64006111-64006170+) in the same chromosome. Thus, there may be a new way that nearby lncRNAs regulate the expression of VEGF-B.

Alhough some lncRNAs are proven as cis-regulators, the vast majority of IncRNAs with known functions are actually trans-regulators (22). In contrast to cis-regulation, the trans-regulatory lncRNAs, after isolation from the synthesis site, affect the genes at a long genomic distance away, accumulate and take effect in large regulatory networks (5). We predicted the functions of trans-regulatory lncRNAs through the relevant expression-regulating TFs. In the core network of lncRNA-TF pairs, the lncRNAs were divided into four groups of pathways that are controlled by E2F4, E2F1, USF1 and TFAP2C, respectively.

E2F4 and E2F1 which belong to the E2F family are proposed to be primary transcription repressors. The E2F 
Table IV. The top 100 lncRNA-TF pairs with the most credentiality.

\begin{tabular}{|c|c|c|c|}
\hline lncRNA & $\mathrm{TF}$ & P-value & FDR \\
\hline NONHSAG029883 & $\mathrm{E} 2 \mathrm{~F} 1$ & 0.000100199 & 0.011522898 \\
\hline NONHSAT098423 & $\mathrm{E} 2 \mathrm{~F} 1$ & 0.00010995 & 0.012644217 \\
\hline FR165245 & $\mathrm{E} 2 \mathrm{~F} 1$ & 0.000114954 & 0.013219731 \\
\hline NONHSAT001522 & $\mathrm{E} 2 \mathrm{~F} 4$ & 0.00011977 & 0.005932082 \\
\hline TCONS_12_00015621 & YY1 & 0.000120282 & 0.013591822 \\
\hline NONHSAG035406 & $\mathrm{E} 2 \mathrm{~F} 1$ & 0.000120559 & 0.013623208 \\
\hline NONHSAT005942 & $\mathrm{E} 2 \mathrm{~F} 1$ & 0.000133523 & 0.015355119 \\
\hline NONHSAT008438 & USF1 & 0.000134804 & 0.007751239 \\
\hline NONHSAT030840 & $\mathrm{E} 2 \mathrm{~F} 4$ & 0.000136262 & 0.010899038 \\
\hline NONHSAG050806 & TFAP2C & 0.000140853 & 0.008099028 \\
\hline NONHSAT122253 & TFAP2C & 0.000141107 & 0.008113627 \\
\hline NONHSAT011117 & USF1 & 0.00014186 & 0.008156953 \\
\hline NONHSAT093783 & $\mathrm{E} 2 \mathrm{~F} 1$ & 0.000148833 & 0.016966919 \\
\hline ENST00000508616 & $\mathrm{E} 2 \mathrm{~F} 1$ & 0.000150861 & 0.017047245 \\
\hline TCONS_00001679 & ATF3 & 0.000153448 & 0.005882192 \\
\hline NONHSAT001522 & $\mathrm{E} 2 \mathrm{~F} 1$ & 0.00015475 & 0.005932082 \\
\hline NONHSAT081186 & USF1 & 0.000163155 & 0.009218242 \\
\hline NR_027459.2 & $\mathrm{E} 2 \mathrm{~F} 1$ & 0.000165025 & 0.018977858 \\
\hline ENST00000420044 & TFAP2C & 0.000165234 & 0.009500979 \\
\hline NONHSAT092847 & $\mathrm{E} 2 \mathrm{~F} 1$ & 0.000170233 & 0.00970326 \\
\hline FR009982 & USF1 & 0.000174482 & 0.02006547 \\
\hline ENST00000598393 & $\mathrm{E} 2 \mathrm{~F} 1$ & 0.000175642 & 0.010099417 \\
\hline NONHSAT028356 & USF1 & 0.000182684 & 0.010504343 \\
\hline ENST00000438810 & TFAP2C & 0.000184836 & 0.010628064 \\
\hline NONHSAT030840 & $\mathrm{E} 2 \mathrm{~F} 1$ & 0.000189548 & 0.010899038 \\
\hline NONHSAT095682 & TFAP2C & 0.000191811 & 0.01102914 \\
\hline NONHSAT129523 & ELK4 & 0.000192126 & 0.022094508 \\
\hline NONHSAT010620 & $\mathrm{E} 2 \mathrm{~F} 1$ & 0.000194595 & 0.02198926 \\
\hline ENST00000533220 & $\mathrm{E} 2 \mathrm{~F} 4$ & 0.000195146 & 0.011123299 \\
\hline NONHSAT017390 & $\mathrm{E} 2 \mathrm{~F} 1$ & 0.000201551 & 0.022372179 \\
\hline NONHSAG001064 & $\mathrm{E} 2 \mathrm{~F} 1$ & 0.000201777 & 0.022800789 \\
\hline NONHSAT140499 & TFAP2C & 0.00020195 & 0.011612109 \\
\hline FR042098 & USF1 & 0.000202339 & 0.011634494 \\
\hline TCONS_12_00021700 & PPARGC1A & 0.000216816 & 0.024283375 \\
\hline NONHSAT120476 & TFAP2C & 0.00022388 & 0.012761147 \\
\hline TCONS_00016368 & $\mathrm{E} 2 \mathrm{~F} 4$ & 0.000243819 & 0.027795322 \\
\hline NONHSAT037766 & ELK4 & 0.000256704 & 0.017378335 \\
\hline NONHSAG031748 & USF1 & 0.000264772 & 0.023070091 \\
\hline FR224199 & $\mathrm{E} 2 \mathrm{~F} 1$ & 0.000266261 & 0.030619965 \\
\hline NONHSAG003987 & TFAP2C & 0.000275049 & 0.015815294 \\
\hline TCONS_12_00020217 & $\mathrm{E} 2 \mathrm{~F} 1$ & 0.000276553 & 0.031250531 \\
\hline NONHSAT090951 & E2F4 & 0.000283269 & 0.032575881 \\
\hline NONHSAT138382 & $\mathrm{E} 2 \mathrm{~F} 1$ & 0.000291224 & 0.032617038 \\
\hline NONHSAT031072 & TFAP2C & 0.00029954 & 0.017223562 \\
\hline NONHSAT118621 & TFAP2C & 0.00029985 & 0.017091435 \\
\hline NONHSAT037766 & $\mathrm{E} 2 \mathrm{~F} 1$ & 0.000302232 & 0.017378335 \\
\hline NONHSAT122928 & TFAP2C & 0.000307854 & 0.017701585 \\
\hline NONHSAT026185 & TFAP2C & 0.000308128 & 0.017717359 \\
\hline ENST00000412387 & E2F4 & 0.000310984 & 0.035763147 \\
\hline ENST00000602721 & TFAP2C & 0.000311238 & 0.017896208 \\
\hline ENST00000434951 & TFAP2C & 0.000318988 & 0.020166596 \\
\hline NR_002834.1 & USF1 & 0.000319364 & 0.018363452 \\
\hline
\end{tabular}


Table IV. Continued.

\begin{tabular}{|c|c|c|c|}
\hline lncRNA & $\mathrm{TF}$ & P-value & FDR \\
\hline NONHSAT030171 & USF1 & 0.00032151 & 0.036652133 \\
\hline NONHSAT035083 & $\mathrm{E} 2 \mathrm{~F} 1$ & 0.000325007 & 0.03672582 \\
\hline NONHSAT060965 & TFAP2C & 0.00032951 & 0.018946816 \\
\hline NONHSAT093783 & $\mathrm{E} 2 \mathrm{~F} 4$ & 0.000334903 & 0.019089467 \\
\hline TCONS_00013626 & E2F1 & 0.00034006 & 0.039106915 \\
\hline NONHSAG039347 & E2F1 & 0.000343688 & 0.039524106 \\
\hline FR314507 & ZBTB7A & 0.000348142 & 0.020018175 \\
\hline NONHSAT022221 & PPARGC1A & 0.000348948 & 0.019715575 \\
\hline ENST00000434951 & TFAP2A & 0.0003538 & 0.020166596 \\
\hline NR_073125.1 & TFAP2C & 0.000355186 & 0.020068009 \\
\hline FR070335 & E2F1 & 0.000360267 & 0.041430707 \\
\hline NONHSAT026990 & USF1 & 0.000375935 & 0.021616259 \\
\hline NONHSAT136810 & TFAP2C & 0.00037671 & 0.021660808 \\
\hline NONHSAT010513 & $\mathrm{E} 2 \mathrm{~F} 1$ & 0.000396964 & 0.045650912 \\
\hline TCONS_00029195 & TFAP2C & 0.000398087 & 0.015818714 \\
\hline NONHSAT119003 & TFAP2C & 0.000403253 & 0.022985416 \\
\hline FR055729 & $\mathrm{E} 2 \mathrm{~F} 1$ & 0.000403717 & 0.0464274 \\
\hline ENST00000417120 & E2F1 & 0.000406492 & 0.045527124 \\
\hline NONHSAG031748 & E2F4 & 0.00040832 & 0.023070091 \\
\hline NONHSAT130274 & TFAP2C & 0.00040865 & 0.023497401 \\
\hline TCONS_00029195 & TFAP2A & 0.000412662 & 0.015818714 \\
\hline NONHSAT096163 & E2F4 & 0.000424883 & 0.024218343 \\
\hline NONHSAT056714 & TFAP2C & 0.000425979 & 0.024493803 \\
\hline NONHSAG037825 & USF1 & 0.000427744 & 0.024595276 \\
\hline NR_104426.1 & TFAP2C & 0.000431261 & 0.024581897 \\
\hline NONHSAT122833 & TFAP2C & 0.000436645 & 0.025107059 \\
\hline TCONS_00027489 & TFAP2C & 0.000442076 & 0.025419365 \\
\hline NONHSAT093875 & USF1 & 0.000443636 & 0.02550908 \\
\hline NONHSAT140507 & E2F1 & 0.00045296 & 0.030884797 \\
\hline NONHSAT023895 & USF1 & 0.000454218 & 0.051326598 \\
\hline NONHSAT077173 & ATF3 & 0.000457842 & 0.026325887 \\
\hline NONHSAT093946 & TFAP2C & 0.000459005 & 0.026392802 \\
\hline NONHSAT119495 & E2F1 & 0.000466442 & 0.053640826 \\
\hline NONHSAT136475 & $\mathrm{E} 2 \mathrm{~F} 4$ & 0.000466481 & 0.026589441 \\
\hline NONHSAT096168 & TFAP2C & 0.000466948 & 0.026616051 \\
\hline NONHSAG046899 & USF1 & 0.000471352 & 0.026867058 \\
\hline NONHSAG050557 & E2F1 & 0.000472068 & 0.05428779 \\
\hline NONHSAT032403 & $\mathrm{E} 2 \mathrm{~F} 1$ & 0.000478104 & 0.054025738 \\
\hline ENST00000561486 & TFAP2C & 0.000479431 & 0.027567279 \\
\hline TCONS_12_00015621 & USF1 & 0.000487468 & 0.02320976 \\
\hline NONHSAT006799 & TFAP2C & 0.000492218 & 0.028302546 \\
\hline NONHSAT034980 & E2F1 & 0.000493572 & 0.056760748 \\
\hline NONHSAT004232 & TFAP2C & 0.000505112 & 0.028791388 \\
\hline NONHSAT079625 & USF1 & 0.000516961 & 0.058933596 \\
\hline NONHSAT095682 & TFAP2A & 0.000525617 & 0.020148659 \\
\hline NONHSAT077174 & TFAP2C & 0.000525879 & 0.024031165 \\
\hline FR280917 & USF1 & 0.00052622 & 0.029994527 \\
\hline TCONS_00005559 & TFAP2A & 0.000534675 & 0.030743841 \\
\hline
\end{tabular}




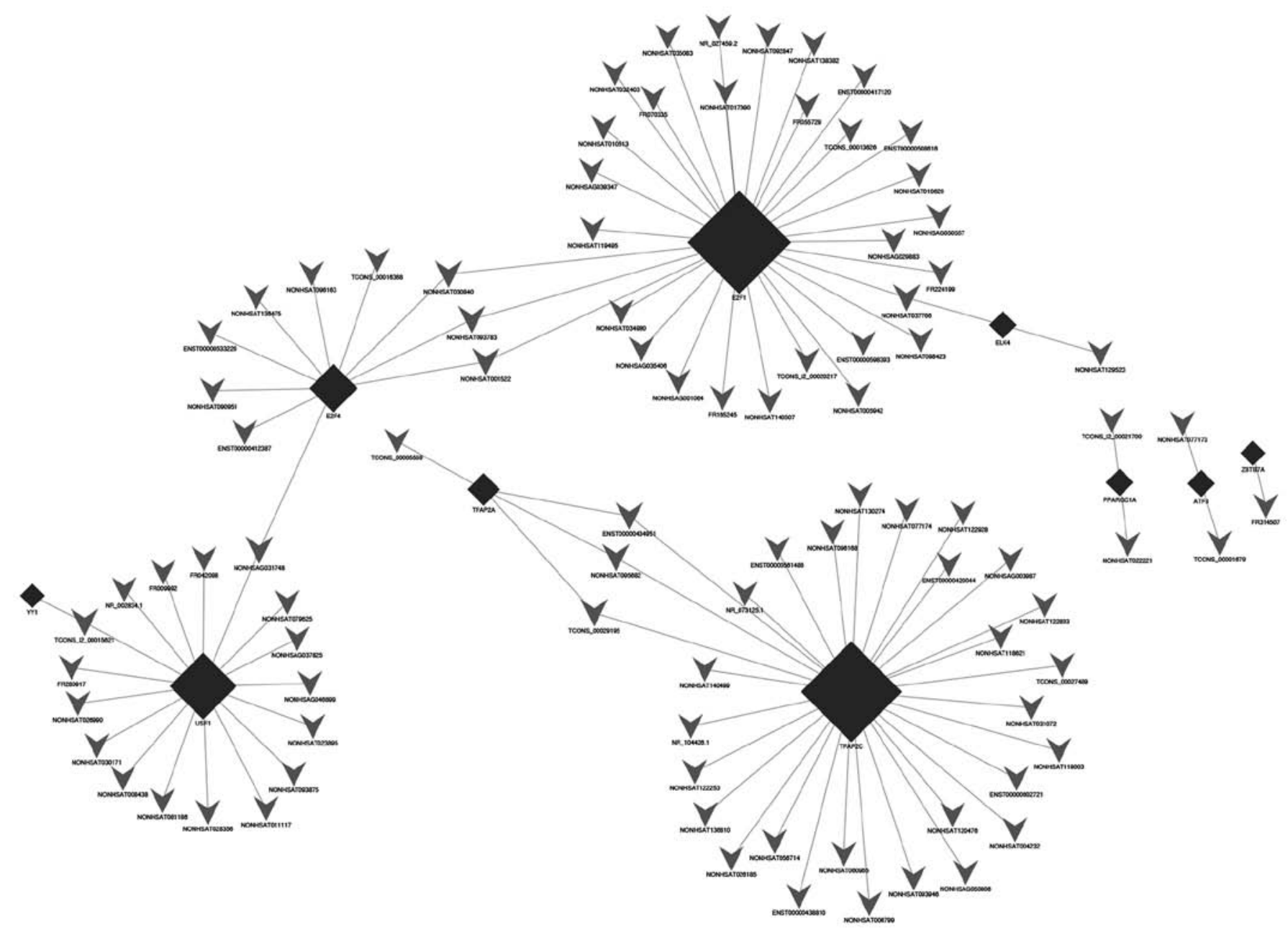

Figure 4. IncRNA-TF core network consisting of the top 100 pairs of lncRNAs and TFs with the most credentiality. Most of the lncRNAs were predicted to be regulated by E2F1, E2F4, USF1 and TFAP2C. The rectangular shapes represent TFs, and arrows represent lncRNAs; the edges between them mean that the IncRNAs are potentially regulated by the TFs.

family is pivotal in the regulation of cell cycle and the action of tumor suppressor proteins. The E2F family is also a target of the transforming proteins of small DNA tumor viruses (23).

E2F1 regulates the transcription of DNA synthesis-related genes and thus, the binding of hypophosphorylated $\mathrm{pRB}$ to E2F1 will arrest G1 phase cells (24). Gefitinib treatment downregulates the expression of E2F1 mRNA and protein. The antiproliferative effect of gefitinib is attributed, or at least partially, to the inhibition of E2F1 expression and increases the proportion of G1 phase cells in human LACs (A431 and A549) (25). E2F1 is also downregulated by gefitinib treatment in gefitinib-resistant NSCLC cells with MET amplification, but not in resistant NSCLC cells harboring the T790M mutation of EGFR (26). The above studies indicate that E2F1 is closely associated with the resistance to EGFR-TKIs. The IncRNAs predicted as regulatory targets of E2F1 may take part in the acquisition of gifitinib resistance, which should be validated by further functional tests.

E2F4 takes part in diverse functions, such as cell cycle control, DNA damage repair, apoptosis, mRNA processing and ubiquitination $(27,28)$. The upregulation of E2F4 nuclear expression in breast cancer is related to poor prognosis in patients with larger tumors, recurrence, distant metastasis and poorer outcome (29). E2F4 protein expression in human colorectal tumors is also upregulated by affecting the G1/S phase transition and the proliferation of colon cancer cells (30). In comparison, E2F4 protein expression is downregulated in sporadic Burkitt lymphoma (sBL) cells but not in immortal B-cells. Besides the ability to reduce E2F1 levels, E2F4 expression in BL cells also selectively weakens the tumorigenic properties and BL cell proliferation and increases the proportions of $\mathrm{G}_{2} / \mathrm{M}$ cells (31). Since E2F4 has diverse effects in the regulation of tumor progression, it may also play a role in lung cancer.

USF1 is a basic helix-loop-helix (bHLH) TF encoded by distinct genes that are heterodimerized and functionally overlapped (32). USF1 also mediates the transcription of many neuropeptides (33-36), surfactant protein A in the lung (37), genes in lung tumors (38) which include hTERT as the immortalizing telomerase subunit $(39,40)$ and CDK4 as the cell cycle regulator (41). USF1 can also regulate neuropeptide genes in lung cancer, particularly arginine vasopressin (AVP) $(42,43)$. USF1 mRNA expression is downregulated in LACs compared with that in normal tissues (44). In lung cancer, USF1 and USF2 dimerize to mediate the transcription via E-box motifs in target genes (45). Moreover, with the E-box motif, USF1 activates 
GATA5 gene expression (46), which is pivotal in cell differentiation and tissue development in lung cancer $(47,48)$. Since USF1 is closely related to lung cancer occurrence, USF1-regulated lncRNAs may be involved in EGFR-TKI resistance.

TFAP2C, also known as AP2- $\gamma$, belongs to the AP-2 TF family, which is composed of five closely related $50-\mathrm{kDa}$ isoforms (49). By regulating the expression of many downstream genes, TFAP2C mediates various cellular processes, including induction, differentiation, survival, proliferation and apoptosis under diverse developmental contexts (50-54). The internalization of activated EGFR involves the AP-2 complex (55). TFAP2C is pivotal in human cancer development. For example, through transcriptional regulation of EGFR, TFAP2C mediates the tumor development, cell growth and survival of HER2-amplified breast cancer (56). Moreover, after 10 years of diagnosis, higher TFAP2C scores are correlated with poor overall survival in ER $\alpha$-positive and endocrine therapy-treated patients (57). Since TFAP2C affects tumorigenesis by mediating EGFR expression, TFAP2C may be an important cause of EGFR-TKI-resistant lung cancer. Thus, the lncRNAs that take part in the TFAP2C-regulated pathways, as predicted, are candidate participants in the mechanisms of EGFR-TKI resistance.

The present study has also a few limitations. Firstly, we only used one cell line to study the mechanisms of EGFR-TKI resistance in vitro. Our results are not integrated but typical, and thus further studies with larger sample size in vivo are needed. Second, we only predicted lncRNA functions indirectly through network and pathway analyses of co-expressed protein-coding genes. The reason is that the majority of the lncRNAs identified to date are not functionally characterized (58). The 'guilt by association' method for hypothesis generation is a key primary step for functional studies in the future, such as loss/acquisition of functions (59).

In conclusion, nearly 1,500 lncRNAs were differently expressed between the gefitinib-sensitive HCC827 cells and the gefitinib-resistant HCC827-8-1 cells. Many of these lncRNAs play important roles in regulating EGFR-TKI resistance through the cis- and/or trans-regulation of target proteincoding genes. The present study underlies future functional studies on lncRNAs related to EGFR resistance and provides a candidate reservoir for their application as diagnostic and therapeutic targets.

\section{Acknowledgements}

The present study was supported by grants from the National Natural Science Foundation of China (81372396).

\section{References}

1. Jackman DM, Miller VA, Cioffredi LA, Yeap BY, Jänne PA, Riely GJ, Ruiz MG, Giaccone G, Sequist LV and Johnson BE: Impact of epidermal growth factor receptor and KRAS mutations on clinical outcomes in previously untreated non-small cell lung cancer patients: Results of an online tumor registry of clinical trials. Clin Cancer Res 15: 5267-5273, 2009.

2. Godin-Heymann N, Bryant I, Rivera MN, Ulkus L, Bell DW, Riese DJ II, Settleman J and Haber DA: Oncogenic activity of epidermal growth factor receptor kinase mutant alleles is enhanced by the T790M drug resistance mutation. Cancer Res 67: 7319-7326, 2007.
3. McDermott U, Pusapati RV, Christensen JG, Gray NS and Settleman J: Acquired resistance of non-small cell lung cancer cells to MET kinase inhibition is mediated by a switch to epidermal growth factor receptor dependency. Cancer Res 70: 1625-1634, 2010.

4. Yamamoto C, Basaki Y, Kawahara A, Nakashima K, Kage M, Izumi H, Kohno K, Uramoto H, Yasumoto K, Kuwano M, et al: Loss of PTEN expression by blocking nuclear translocation of EGR1 in gefitinib-resistant lung cancer cells harboring epidermal growth factor receptor-activating mutations. Cancer Res 70: 8715-8725, 2010.

5. Lee JT: Epigenetic regulation by long noncoding RNAs. Science 338: 1435-1439, 2012.

6. Qiu M, Xu Y, Yang X, Wang J, Hu J, Xu L and Yin R: CCAT2 is a lung adenocarcinoma-specific long non-coding RNA and promotes invasion of non-small cell lung cancer. Tumour Biol 35: 5375-5380, 2014.

7. Gutschner T, Hämmerle M, Eissmann M, Hsu J, Kim Y, Hung G, Revenko A, Arun G, Stentrup M, Gross M, et al: The noncoding RNA MALAT1 is a critical regulator of the metastasis phenotype of lung cancer cells. Cancer Res 73: 1180-1189, 2013.

8. Liu XH, Liu ZL, Sun M, Liu J, Wang ZX and De W: The long non-coding RNA HOTAIR indicates a poor prognosis and promotes metastasis in non-small cell lung cancer. BMC Cancer 13: 464, 2013.

9. Liu Z1, Sun M, Lu K, Liu J, Zhang M, Wu W, De W, Wang Z and Wang R: The long noncoding RNA HOTAIR contributes to cisplatin resistance of human lung adenocarcinoma cells via downregualtion of $\mathrm{p} 21^{\mathrm{WAF} / \mathrm{CIP} 1}$ expression. PLoS One 8: e77293, 2013.

10. Dong S, Qu X, Li W, Zhong X, Li P, Yang S, Chen X, Shao M and Zhang L: The long non-coding RNA, GAS5, enhances gefitinib-induced cell death in innate EGFR tyrosine kinase inhibitor-resistant lung adenocarcinoma cells with wide-type EGFR via downregulation of the IGF-1R expression. J Hematol Oncol 8: 43, 2015.

11. Wu Y, Yu DD, Hu Y, Cao HX, Yu SR, Liu SW and Feng JF: LXR ligands sensitize EGFR-TKI-resistant human lung cancer cells in vitro by inhibiting Akt activation. Biochem Biophys Res Commun 467: 900-905, 2015.

12. Livak KJ and Schmittgen TD: Analysis of relative gene expression data using real-time quantitative PCR and the $2^{-\Delta \Delta C_{\mathrm{T}}}$ method. Methods 25: 402-408, 2001.

13. Guttman M, Amit I, Garber M, French C, Lin MF, Feldser D, Huarte M, Zuk O, Carey BW, Cassady JP, et al: Chromatin signature reveals over a thousand highly conserved large noncoding RNAs in mammals. Nature 458: 223-227, 2009.

14. Efron B and Tibshirani R: Empirical bayes methods and false discovery rates for microarrays. Genet Epidemiol 23: 70-86, 2002.

15. Guttman M, Donaghey J, Carey BW, Garber M, Grenier JK, Munson G, Young G, Lucas AB, Ach R, Bruhn L, et al: lincRNAs act in the circuitry controlling pluripotency and differentiation. Nature 477: 295-300, 2011.

16. Remon J, Morán T, Majem M, Reguart N, Dalmau E, MárquezMedina D and Lianes P: Acquired resistance to epidermal growth factor receptor tyrosine kinase inhibitors in $E G F R$ mutant non-small cell lung cancer: A new era begins. Cancer Treat Rev 40: 93-101, 2014.

17. Li LH, Wu P, Lee JY, Li PR, Hsieh WY, Ho CC, Ho CL, Chen WJ, Wang CC, Yen MY, et al: Hinokitiol induces DNA damage and autophagy followed by cell cycle arrest and senescence in gefitinibresistant lung adenocarcinoma cells. PLoS One 9: e104203, 2014.

18. Ju L and Zhou C: Association of integrin beta1 and c-MET in mediating EGFR TKI gefitinib resistance in non-small cell lung cancer. Cancer Cell Int 13: 15, 2013.

19. Terai H, Soejima K, Yasuda H, Sato T, Naoki K, Ikemura S, Arai D, Ohgino K, Ishioka K, Hamamoto J, et al: Long-term exposure to gefitinib induces acquired resistance through DNA methylation changes in the EGFR-mutant PC9 lung cancer cell line. Int J Oncol 46: 430-436, 2015.

20. Ørom UA, Derrien T, Beringer M, Gumireddy K, Gardini A, Bussotti G, Lai F, Zytnicki M, Notredame C, Huang Q, et al: Long noncoding RNAs with enhancer-like function in human cells. Cell 143: 46-58, 2010.

21. Yang X, Zhang Y, Hosaka K, Andersson P, Wang J, Tholander F, Cao Z, Morikawa H, Tegnér J, Yang Y, et al: VEGF-B promotes cancer metastasis through a VEGF-A-independent mechanism and serves as a marker of poor prognosis for cancer patients. Proc Natl Acad Sci USA 112: E2900-E2909, 2015. 
22. Guttman M and Rinn JL: Modular regulatory principles of large non-coding RNAs. Nature 482: 339-346, 2012.

23. Vaishnav YNV, Vaishnav MY and Pant V: The molecular and functional characterization of E2F-5 transcription factor. Biochem Biophys Res Commun 242: 586-592, 1998.

24. Stewart ZA, Westfall MD and Pietenpol JA: Cell-cycle dysregulation and anticancer therapy. Trends Pharmacol Sci 24: 139-145, 2003.

25. Suenaga M, Yamaguchi A, Soda H, Orihara K, Tokito Y, Sakaki Y, Umehara M, Terashi K, Kawamata N, Oka M, et al: Antiproliferative effects of gefitinib are associated with suppression of E2F-1 expression and telomerase activity. Anticancer Res 26: 3387-3391, 2006.

26. Okabe T, Okamoto I, Tsukioka S, Uchida J, Hatashita E, Yamada Y, Yoshida T, Nishio K, Fukuoka M, Jänne PA, et al: Addition of S-1 to the epidermal growth factor receptor inhibitor gefitinib overcomes gefitinib resistance in non-small cell lung cancer cell lines with MET amplification. Clin Cancer Res 15: 907-913, 2009.

27. Lindeman GJ, Gaubatz S, Livingston DM and Ginsberg D: The subcellular localization of E2F-4 is cell-cycle dependent. Proc Natl Acad Sci USA 94: 5095-5100, 1997.

28. Mikkelsen TS, Ku M, Jaffe DB, Issac B, Lieberman E, Giannoukos G, Alvarez P, Brockman W, Kim TK, Koche RP, et al: Genome-wide maps of chromatin state in pluripotent and lineage-committed cells. Nature 448: 553-560, 2007.

29. Rakha EA, Pinder SE, Paish EC, Robertson JF and Ellis IO: Expression of E2F-4 in invasive breast carcinomas is associated with poor prognosis. J Pathol 203: 754-761, 2004.

30. Garneau H, Paquin MC, Carrier JC and Rivard N: E2F4 expression is required for cell cycle progression of normal intestinal crypt cells and colorectal cancer cells. J Cell Physiol 221: 350-358, 2009

31. Molina-Privado I, Jiménez-P R, Montes-Moreno S, Chiodo Y, Rodríguez-Martínez M, Sánchez-Verde L, Iglesias T, Piris MA and Campanero MR: E2F4 plays a key role in Burkitt lymphoma tumorigenesis. Leukemia 26: 2277-2285, 2012.

32. Sirito M, Lin Q, Deng JM, Behringer RR and Sawadogo M: Overlapping roles and asymmetrical cross-regulation of the USF proteins in mice. Proc Natl Acad Sci USA 95: 3758-3763, 1998.

33. Nielsen FC, Pedersen K, Hansen TV, Rourke IJ and Rehfeld JF: Transcriptional regulation of the human cholecystokinin gene: Composite action of upstream stimulatory factor, Sp1, and members of the CREB/ATF-AP-1 family of transcription factors. DNA Cell Biol 15: 53-63, 1996.

34. Viney TJ, Schmidt TW, Gierasch W, Sattar AW, Yaggie RE, Kuburas A, Quinn JP, Coulson JM and Russo AF: Regulation of the cell-specific calcitonin/calcitonin gene-related peptide enhancer by USF and the Foxa2 forkhead protein. J Biol Chem 279: 49948-49955, 2004.

35. Paterson JM, Morrison CF, Mendelson SC, McAllister J and Quinn JP: An upstream stimulatory factor (USF) binding motif is critical for rat preprotachykinin-A promoter activity in PC12 cells. Biochem J 310: 401-406, 1995.

36. Hadsell DL, Bonnette S, George J, Torres D, Klimentidis Y, Gao S, Haney PM, Summy-Long J, Soloff MS, Parlow AF, et al: Diminished milk synthesis in upstream stimulatory factor 2 null mice is associated with decreased circulating oxytocin and decreased mammary gland expression of eukaryotic initiation factors 4E and 4G. Mol Endocrinol 17: 2251-2267, 2003.

37. Gao E, Wang Y, Alcorn JL and Mendelson CR: Transcription factor USF2 is developmentally regulated in fetal lung and acts together with USF1 to induce $S P$ - $A$ gene expression. Am J Physiol Lung Cell Mol Physiol 284: L1027-L1036, 2003.

38. Coulson JM, Edgson JL, Marshall-Jones ZV, Mulgrew R, Quinn JP and Woll PJ: Upstream stimulatory factor activates the vasopressin promoter via multiple motifs, including a noncanonical E-box. Biochem J 369: 549-561, 2003.

39. McMurray HR and McCance DJ: Human papillomavirus type 16 E6 activates TERT gene transcription through induction of c-Myc and release of USF-mediated repression. J Virol 77: 9852-9861, 2003.

40. Goueli BS and Janknecht R: Regulation of telomerase reverse transcriptase gene activity by upstream stimulatory factor Oncogene 22: 8042-8047, 2003.
41. Pawar SA, Szentirmay MN, Hermeking H and Sawadogo M: Evidence for a cancer-specific switch at the $C D K 4$ promoter with loss of control by both USF and c-Myc. Oncogene 23: 6125-6135, 2004.

42. Coulson JM, Fiskerstrand CE, Woll PJ and Quinn JP: E-box motifs within the human vasopressin gene promoter contribute to a major enhancer in small-cell lung cancer. Biochem J 344: 961-970, 1999

43. Grace CO, Fink G and Quinn JP: Characterization of potential regulatory elements within the rat arginine vasopressin proximal promoter. Neuropeptides 33: 81-90, 1999.

44. Khattar NH, Lele SM and Kaetzel CS: Down-regulation of the polymeric immunoglobulin receptor in non-small cell lung carcinoma: Correlation with dysregulated expression of the transcription factors USF and AP2. J Biomed Sci 12: 65-77, 2005.

45. Ocejo-Garcia M, Baokbah TA, Ashurst HL, Cowlishaw D, Soomro I, Coulson JM and Woll PJ: Roles for USF-2 in lung cancer proliferation and bronchial carcinogenesis. J Pathol 206: 151-159, 2005.

46. Chen B, Hsu R, Li Z, Kogut PC, Du Q, Rouser K, CamorettiMercado B and Solway J: Upstream stimulatory factor 1 activates GATA5 expression through an E-box motif. Biochem J 446: 89-98, 2012.

47. Kakita T, Hasegawa K, Morimoto T, Kaburagi S, Wada H and Sasayama S: p300 protein as a coactivator of GATA-5 in the transcription of cardiac-restricted atrial natriuretic factor gene. J Biol Chem 274: 34096-34102, 1999.

48. Singh MK, Li Y, Li S, Cobb RM, Zhou D, Lu MM, Epstein JA, Morrisey EE and Gruber PJ: Gata4 and Gata5 cooperatively regulate cardiac myocyte proliferation in mice. J Biol Chem 285: $1765-1772,2010$

49. Tummala R, Romano RA, Fuchs E and Sinha S: Molecular cloning and characterization of AP-2 epsilon, a fifth member of the AP-2 family. Gene 321: 93-102, 2003.

50. Hoffman TL, Javier AL, Campeau SA, Knight RD and Schilling TF: Tfap2 transcription factors in zebrafish neural crest development and ectodermal evolution. J Exp Zoolog B Mol Dev Evol 308: 679-691, 2007.

51. Kuckenberg P, Kubaczka $\mathrm{C}$ and Schorle H: The role of transcription factor Tcfap2c/TFAP2C in trophectoderm development. Reprod Biomed Online 25: 12-20, 2012.

52. Li W and Cornell RA: Redundant activities of Tfap2a and Tfap $2 \mathrm{c}$ are required for neural crest induction and development of other non-neural ectoderm derivatives in zebrafish embryos. Dev Biol 304: 338-354, 2007.

53. Li X, Glubrecht DD and Godbout R: AP2 transcription factor induces apoptosis in retinoblastoma cells. Genes Chromosomes Cancer 49: 819-830, 2010.

54. Van Otterloo E, Li W, Garnett A, Cattell M, Medeiros DM and Cornell RA: Novel Tfap2-mediated control of soxE expression facilitated the evolutionary emergence of the neural crest. Development 139: 720-730, 2012.

55. Rappoport JZ and Simon SM: Endocytic trafficking of activated EGFR is AP-2 dependent and occurs through preformed clathrin spots. J Cell Sci 122: 1301-1305, 2009.

56. Park JM, Wu T, Cyr AR, Woodfield GW, De Andrade JP, Spanheimer PM, Li T, Sugg SL, Lal G, Domann FE, et al: The role of Tcfap $2 \mathrm{c}$ in tumorigenesis and cancer growth in an activated Neu model of mammary carcinogenesis. Oncogene 34: 6105-6104, 2015

57. Perkins SM, Bales C, Vladislav T, Althouse S, Miller KD, Sandusky G, Badve S and Nakshatri H: TFAP2C expression in breast cancer: Correlation with overall survival beyond 10 years of initial diagnosis. Breast Cancer Res Treat 152: 519-531, 2015.

58. Derrien T, Johnson R, Bussotti G, Tanzer A, Djebali S, Tilgner H, Guernec G, Martin D, Merkel A, Knowles DG, et al: The GENCODE v7 catalog of human long noncoding RNAs: Analysis of their gene structure, evolution, and expression. Genome Res 22: 1775-1789, 2012.

59. Rinn JL and Chang HY: Genome regulation by long noncoding RNAs. Annu Rev Biochem 81: 145-166, 2012. 